\title{
ENHANCEMENT OF EXTERNAL WALL DECORATION MATERIAL FOR THE BUILDING IN SAFETY INSPECTION METHOD
}

\author{
Nai-Hsin PAN ${ }^{1}$, Ching-Hsiang TSAI ${ }^{2}$, Kuei-Yuan CHEN ${ }^{1}$, Jessie $\mathrm{SUNG}^{3 *}$ \\ ${ }^{1}$ Department of Civil and Construction Engineering, National Yunlin University \\ of Science and Technolog, Douliu, R.O.C. \\ ${ }^{2}$ Graduate School of Engineering Science and Technology, National Yunlin University \\ of Science and Technology, Douliu, R.O.C. \\ ${ }^{3}$ Office of Huamn Resources, Chaoyang University of Technology, Taichung, R.O.C.
}

Received 05 April 2019; accepted 04 September 2019

\begin{abstract}
As buildings wear out, external wall tiles or attachments will usually fall off, sometimes causing human injuries. At present, the method employed for middle-high rise buildings is mainly the method of visual inspection. The inspection results in using this method are affected by the factors of subjectivity, safety and cost. This study aims to provide a lowercost and more efficient evaluation method for inspecting the status of buildings' external walls. This proposed method implements Forward Looking Infrared (FLIR) technology and high-resolution photographic equipment on Unmanned Aerial Vehicle (UAV) which can improve the image recording of the detection process, as well as the overall visual detection technology, and solve the existing visual detection problem of inspectors. Also, the images detected by visual inspection and UAV high-resolution video are used to develop a suitable visual evaluation process and test table for external walls. Through the test results of several cases, the deterioration status and needs for maintenance are taken into account according to the degree of performance indicators. The findings of the study is that the proposed mechanism is more efficient and lower cost for the detection of external walls or ancillary structures' abnormal status, which is easy to use in practice.
\end{abstract}

Keywords: UAV, infrared thermal imager, external walls, buildings, maintenance management, detection method.

\section{Introduction}

As buildings wear out, external wall tiles or attachments will usually fall off, sometimes causing human injuries. The competent authorities have established regular inspection mechanisms for the external walls of buildings of a certain age. At present, the method employed for middlehigh rise buildings is mainly visual inspection. Inspectors set up eagles on buildings and inspect them through visual inspection; however, the inspection results in using this method are affected by subjective factors. In addition, the hanging detection that is often used can prevent inspectors from falling; however, the operating cost is not cheap using the method with scaffold. In addition, while telescopes are often used for detection, their effectiveness is limited by height and angle, and inspectors cannot make clear judgments when they are standing atop the building and looking downwards. These techniques for external wall detection have their respective strengths and weaknesses. Therefore, this study aims to provide a lower-cost and more efficient evaluation method for inspecting the status of buildings' external walls. This method is widely used; it mainly implements Forward Looking Infrared (FLIR) technology and high-resolution photographic equipment on Unmanned Aerial Vehicle (UAV). With the maturity of UAV technology and the convenience of operation, the function of the Global Positioning System (GPS) in UAVs can provide the location of detected points and even the setting of relevant height more effectively. Its stability can improve the image recording of the detection process, as well as the overall visual detection technology, and solve the existing visual detection problem of inspectors unable to view from top to bottom at certain angles or when there are shelters.

The use of UAV equipped with high-resolution lens, combined with auxiliary image recording provided by FLIR, can significantly improve the accuracy of visual inspection because this technology can produce images

*Corresponding author. E-mail: sfsung@cyut.edu.tw 
of temperature differentiation in bumps or cracks. Therefore, the use of high-resolution photographic recording equipment in building spare parts can overcome problems caused by the top-down view, angle, temperature and altitude; in addition, the cost is relatively low. In the subsequent processing of computer image comparison and interpretation, the relevant CAD design drawings and grading can be used to assist in interpretation. This method can reduce accidents at work in consideration of public safety, and the cost of providing temporary facilities.

This study aims to propose a more efficient and lowercost detection method that improves the accuracy of current inspection, and that effectively compares the results of deterioration of exterior walls or ancillary structures to grasp their characteristics and trends and properly assess, diagnose and tackle problems. When exterior walls have deteriorated or during routine maintenance, assessment must be performed as routine quality inspection and longterm follow-up inspection. Therefore, the objectives of this study are as follows:

1. A close-range detection method based on visual detection and UAV is proposed, with an evaluation on whether up-down inspection is possible when shelters exist.

2. Whether FLIR can be added to a UAV-based GPS positioning system to enhance the images of temperature differentiation to assist in interpretation, increase the accuracy of visual detection and enable a case study will be discussed.

3. The image comparison method is proposed to compare the original CAD layers of buildings with UAV images for short-range detection and simulation, and to estimate the size and range of damaged patterns and areas.

4. The information detected by visual inspection and UAV high-resolution video is used to develop a suitable visual evaluation process and test table for external walls. Through the test results of several cases, the deterioration status and needs for maintenance are taken into account according to the degree of performance indicators.

\section{Literature review}

The section will explore the literature related to external wall inspection and the use of UAVs to integrate the strategies of application and maintenance.

\subsection{Structural surface detection}

Most previous studies on inpection technology are related to materials and non-destructive testing. Lee et al. (2014) used infrared thermography to test the bonding defects of exterior wall decorative tiles of buildings as well as the bonding quality of exterior wall decorative tiles, in order to determine the boundaries of empty drum, calculate the rate of empty drum and analyze the influencing factors and maintenance schemes. The analysis of a pull-out test and non-destructive testing method of bonding strength of exterior wall decorative tiles by Chiao and Kao (2015) provides a theoretical basis for future engineering practice. Yeh (2014) evaluated the quality of the exterior wall of an existing building by analyzing the causes of damage and proposed suggestions for subsequent renovation. Tai (2008) proposed different sticking methods and used the sound-tapping method to detect the sticking condition of tiles of the RC wall structure; it shows that the sound frequency measured by sound-tapping method was digitized and mapped through fast Fourier transform. Yen (2013) proposed that the frequency domain can be easily misjudged due to the poor material quality. Therefore, this study proposes the sound energy area method based on time domain, without the above restrictions, as the basis for judging the deterioration of tiles. Furthermore, in order to collect consistent knock energy samples, the sound energy patterns of heterogeneous tones and normal tones are compared through sound analysis software. The area of normal tones is calculated through the area of sound energy burst peaks using the above-mentioned judgment mode. After verification, the parameters selected in this study can differentiate the heterogeneous tones from normal tones to simulate inspection classification and the quality management method: Grade 1 (red), Grade 2 (yellow), Grade 3 (green). Lin (2014) applied FLIR thermal imaging technology to ordinary exterior wall tiles to detect the debonding between surface tiles, bonding layer and bottom concrete, given that the air layer produced by debonding changes the temperature between normal and defective areas of exterior wall under sunshine. The difference can be judged by real-time display of infrared thermal images. However, this detection method can only indicate the pre-warning of defects without pointing out the interface location of defects. FLIRthermal imaging technology is used to detect the bonding and influencing factors of the bonding layer material of the external wall tile system and the active heating test body to obtain the diachronic temperature curve of the surface. The thermal image and curve in the process of heating can clearly locate the simulated debonding defect embedded in the test body, which confirms the feasibility of this technology. Edis et al. (2013) adopted the FLIR thermal imaging and quasi-quantization method based on time-dependent infrared thermal imaging detection to record and analyze temperature. Through the analysis of the quantitative method, humidity rise has been determined in the place where the ceramic external wall is bonded. Simple image subtraction (SIS), non-negative matrix factorization (NMF) and principal component analysis (PCA) have been used to test and analyze infrared images, and auxiliary techniques, such as pounding for control and surface humidity measurement, are used to assess the results. According to de Freitas et al. (2014), infrared thermal imaging can provide non-destructive diagnosis and be applied to laboratory and field-testing to assess the presentation of spalling results through dynamic thermal imaging and 
to compare with numerical simulation results. The flaws result from radiation through sunlight or heat sources, indicating that once these flaws appear between gypsum and masonry, they lead to additional thermal resistance that changes the abnormal distribution of temperature and air layer in the area. Chen et al. (2015) proposed a 3D object tracking method using image sets and depth-based shelter detection, each of which includes training and testing examples. This paper first represents each image set as its natural second-order statistical data and uses Kernel Partial Least Squares (KPLS) to self-adaptively learn the object representation of the low-dimensional discriminant feature subspace. To prevent occlusion in the process of updating an improper appearance model, this paper uses the detection occlusion information obtained by binocular vision to alleviate the drift of tracking caused by model updating. Huang et al. (2010) applied the concept of D.E.R.U. visual inspection criteria and constructed a set of "visual inspection and evaluation methods for the public safety of building exterior walls" by using the hierarchical analysis method (AHP) and the questionnaire for experts and scholars that contributed factors and weight for the public security inspection required for building exterior walls. Cha et al. (2017) applied the IPTs implemented for detecting civil infrastructure defects to partially replace human-conducted onsite inspections. Cha et al. (2018) applied Computer vision-based techniques, which were developed to overcome the limitations of visual inspection by trained human resources and to remotely detect structural damage in images. BeckMan et al. (2019) proposed a faster region-based convolutional neural network (Faster R-CNN)-based concrete spalling damage detection method with an inexpensive depth sensor to simultaneously quantify multiple instances of spalling in the same surface separately while considering multiple surfaces in structural elements.

\subsection{UAV application}

The UAV refers to the flying vehicle whose flight radius is beyond sight. The great improvements made from 1980 to 2010 made the today's UAV possible, particularly in the following four areas:

1. GPS. In 1981, the first commercial-purpose GPS receiver weighed 50 pounds and its price was as high as USD 100,000 . Now GPS weighs only 0.3 grams and the chip price was less than USD $\$ 5$.

2. IMU. Inertial Measurement Unit (IMU) is used to measure the speed, azimuth and acceleration of UAVs. An IMU (think about Project Apollo) weighed 50 pounds and cost millions of dollars in the1960s.

3. Digital camera. In 1976, Kodak launched the first digital camera with only 100,000 pixels, weighing 3.75 pounds and priced over USD 10,000. The digital camera today is significantly different from that of 1976 (1,000 times higher resolution, 1,000 times smaller size and 100 times lower price).
4. Computer and wireless communication (Wi-Fi, Bluetooth). Undoubtedly, the cost-effectiveness ratio of computers and wireless communication is notably (almost a billion times) better than that in the 1980s.

UAV can be applied to the following areas:

1. Agriculture: UAVs monitor disasters and collect real-time data on crop health and yield.

2. Energy: energy companies use UAVs to monitor pipelines and rigs.

3. Real estate and architecture: UAVs take photos of sites such as golf courses, skyscrapers, and target sites, informing property developers, and monitoring the progress of projects.

4. Rapid response and emergency services: UAVs use infrared sensors to assist in search and rescue operations in a range of scenarios, such as firefighting, ruins and avalanches.

5. News: UAVs report breaking news/disaster/war zone situations in a quicker and safer manner than humans do.

Recently, the remarkable development of the quality of low-cost digital cameras and close-range photogrammetry technology, like the application of computer vision technology (motion structure), has made significant progress such as photogrammetry software (Fonstad et al., 2013; Green et al., 2014). Simultaneously, lightweight RPAS (Remote Pilot Systems) has been equipped with increasingly advanced and reliable navigation systems (Nex \& Remondino, 2014; Turner et al., 2012; Mancini et al., 2013). The mixture of these two technological developments has led to the rapid and widespread use of lightweight RPAS in photogrammetry, especially for cultural heritage (Hashim et al., 2012). Therefore, more and more technicians and professionals from various fields, which are not strictly in the area of measurement, have begun to apply the digital photogrammetry technology of RPAS. This trend points to the need for simple guidelines for the proper use of lowcost photogrammetry performed from very light RPAS (less than $2 \mathrm{~kg}$ ) to obtain a sufficiently accurate threedimensional model of the object being measured (Koutsoudis et al., 2014).

The emergence of UAV cameras can make up for the current drawbacks; they capture clear pictures and possess wide vision by using the concept of gimbal, that can be traced back more than 200 years. Gimbal technology was well known in the 15th century, featuring a pivotal device that enables objects to rotate around a single axis without bias. Each three vertical pivot axes that are vertical with each other constitute a gimbal group, so that the object in the innermost part of the frame can maintain the position of spin axis unchanged. In the early days, the gimbal was mainly used in navigation. The crew would place the compass on the gimbal so that the compass would not be affected when ships oscillated. Currently, the gimbal is not only used for navigation, but also for photography. When users take videos, the gimbal is fixed on the camera to keep the picture stable, as shown in Figure 1. 


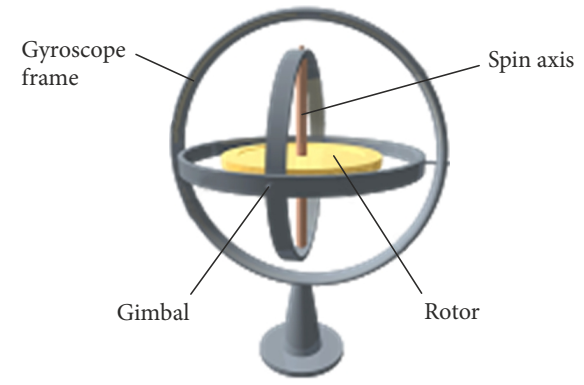

Figure 1. Structure of a gimbal

The UAV gimbal is mainly powered by the engine that controls each axis. When users are surfing in the ocean or skiing, the camera lens automatically captures pictures as long as the camera with a route set is installed on UAV. In addition, when the camera moves, the computer program determines whether it is under user instruction or simply oscillates, so that the image can be maintained at a benchmark point and be prevented from shaking due to external forces. The disadvantage of UAV is the huge power consumption that requires users to prepare extra batteries at any time (Technews, n.d.).

However, the standard operation mode of any UAV should include at least default route, autonomous driving and satellite navigation. That is to say, after taking off by remote control, the aircraft can automatically fly on the route input into the flight computer in advance, modify the flight route by verifying coordinates through the GPS on the aircraft, and transmit flight information back to the ground control center. After the UAV arrives at the target area, ground controllers perform scheduled tasks by remotely controlling the equipment on UAV aircraft via radio. Engel et al. (2014) used a monocular camera as the main transmitter, so no external tracking tool such as GPS or visual tags was needed. The costly computing includes an external laptop and the four-axis UAV of a wireless local area network. The method proposed in this study consists of three components: a monocular SLAM system, extended Kalman filter data fusion, and a PID controller. This is a novel method for estimating the single-eye SLAM system of the transmitter and making the flight accuracy and agility of the external motion capture system possible. Bošnak's et al. (2012) UAV research focuses on vehicle positioning that aims at achieving the self-control of the external transmitter of a fully automatic UAV. If the global positioning system (GPS) fails to work, computerized vision is needed to enable a four-axis aircraft to stabilize its position via the visual system. This marks the development of data fusion algorithms for measuring and positioning by using inertial sensors and visual systems. Hehn and D'Andrea (2014) suggested that UAVs provide applications for aerial robots given the potential of hovering ability and the large dynamic platform. Since the modeling has proven difficult, the control of algorithms usually relies on simplified models to feedback, correct and compensate for non-modeling effects, which may lead to significant tracking errors and repeated execution in high- performance flight. Tracking errors are mostly caused by repeated execution. Kang and Cha (2018) propose an autonomous UAV method using ultrasonic beacons to replace the role of GPS, a deep convolutional neural network $(\mathrm{CNN})$ for damage detection, and a geo-tagging method for the localization of damage.

Therefore, based on the above, this study aims to introduce a more efficient and lower cost mechanism of visual inspection image comparison using the UAV's imager and FLIR thernmal imaging shooting to the detection of external walls or ancillary structures' abnormal status in practice; the feasibility and applicability of the detection methods are discussed. The classification of detection and the degree of detection results are distinguished, and appropriate maintenance methods are selected to repair or renovate buildings, and then several new detection cases are selected for verification. The method proposed in this study can enhance the efficiency of safety and convenience of inspection personnel at a lower cost.

\section{Research method}

This study mainly aims to propose a modified inspection method of external wall that combines UAV (model DJIPhantom 3 Standard) with infrared thermal imager (FLIR, model C2) thermal imaging recording, and case studies to explore the applicability of the proposed method; expert interviews are conducted to improve the application of the detection technology. The proposed method is mainly characterized by two technologies used in the detection process. Firstly, the proposed method implements the infrared thermal imager used to identify the possible abnormal positions at a wider range of temperature chromatic aberrations. It is used in the initial detection in the proposed method; however, implementing the technology should consider the factors of height and shielding which will affect the detection results. Secondly, the proposed method implements UAV, which is mainly applied for close-up recording of the deterioration status of high-rise buildings' external walls. The procedures of this proposed method (Figure 2) are described as follows:

Step 1: Select the detected target and make a preliminary judgment by visually observing the target to select a position with fewer obstacles in that area.

Step 2: After selecting the detection range of the object, firstly, use the infrared camera to record a wide range of images' data which can be used for photo overlay.

Step 3: After inspecting the images from infrared camera from above steps, select and record the suspicious positions in the impages according to the temperature chromatic aberration. Use the UAV for close-range shooting of the positions. Since the UAV has the functions of GPS positioning and hovering, it helps to produce images with higher resolution at higher altitude and close range.

Step 4: Inspect the images captured by the UAV. If there are images that do not identify, take photos again if necessary. 


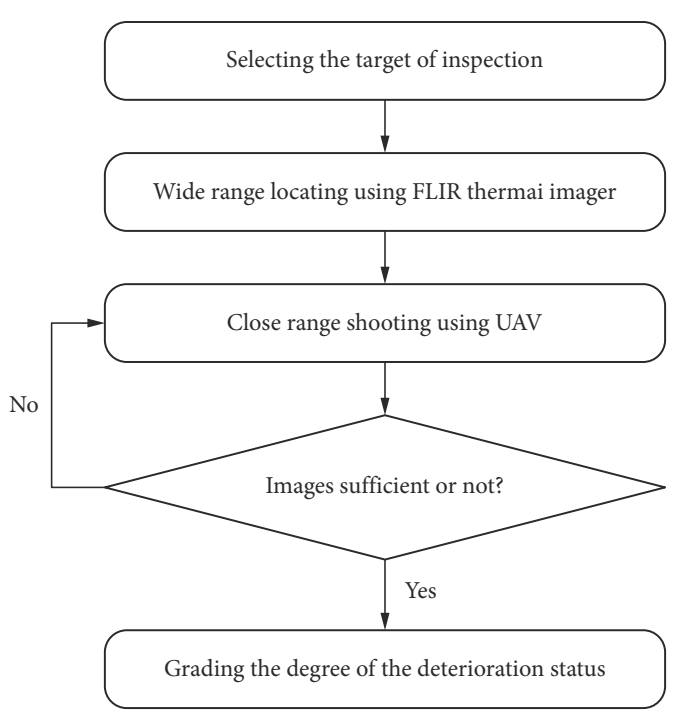

Figure 2. The process of the proposed model

Step 5: After collecting the images of the target through the above methods, proceed with the visual evaluation of the targets' status from those images.

The proposed method mainly implements a UAV with an equipped camera whose images from aerial photography by UAV are transmitted to mobile devices in a wireless and real-time manner and recorded in the memory card of the camera synchronously. Large-area images are recorded by the FLIR thermal imager, followed by highresolution images for inspection and interpretation. After the target is inspected through image comparison, inspectors can evaluate the degree of the targets' damage. In addition, the proposed method evaluates and records the data of external walls' current status and adjacent structures for future maintenance decision making strategies.

\subsection{Application of the FLIR thermal imager}

The FLIR thermal imager uses photoelectric technology to detect specific infrared band signals of thermal radiation of targets, which can be converted into image patterns that can be visualized by humans, and the temperature value can be further calculated. This technology can detect the temperature distribution on the surface of objects (as shown in Figure 3). It features contactlessness, fast temperature measurement and direct visual observation. Therefore, it can be used for non-destructive tests such as for building and bridge structure inspection to detect problems in advance. A FLIR thermal imager has a wide range of applications, including the detection of cracks in buildings, abnormal parts of walls, leakage in walls and underground pipelines, the heat consumption and heating of buildings, and the maintenance and diagnosis of insulation and lighting systems and bridge structure (Lee, 2015).

The advantages and disadvantages of the FLIR thermal imaging inspection and its scope of application after collecting and collecting relevant literature review are described as follows:

- Advantages: the method is a passive technology that does not require a heat source and can be used all day, which is suitable for detecting hot or cold spots. It can penetrate the smoke with its visible light generated from thermal radiation, results displayed instantly, can be used for non-destructive test, and is suitable for remote operation. Currently, the detection speed of the instrument is faster than the traditional temperature measurement method; it can measure or control the temperature instantly. The pattern of heat distribution using the technique can be observed and evaluated.

- Disadvantages: it is unsuitable for conditions of complicated detection (such as internal detection of the target) and emissivity problems. In addition, there could be reflection problems: large area heating is not easy to be uniform with the characteristics of active detection and is more suitable for shallow defect detection. The personnel using the technique need to be equipped wih more related experience.

- Applications: electrical products with possible contact risk, fly contact measurement, remote target (unreachable), large-area target, large-scale measurement.

\subsection{Discussion of UAV image recording application}

The aerial images taken by a UAV's camera aim at providing closer and faster viewing compared with the traditional visual inspection method. Furthermore, the proposed
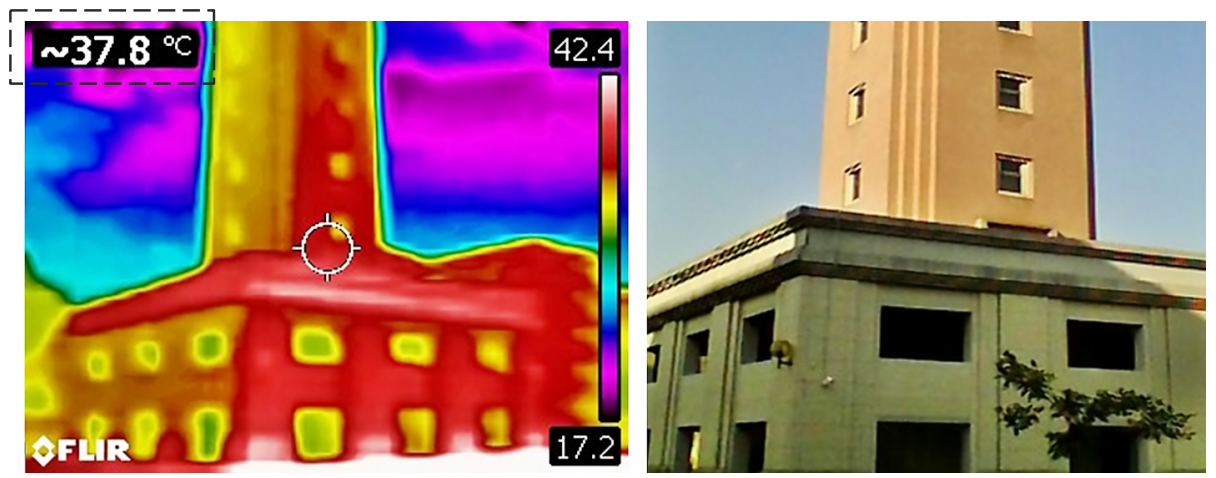

Figure 3. Infrared thermal imager detection case: detection of exterior wall peeling (infrared and visible images) 
method can evaluate the overall or structural fixed-point locations and simplify the inspection process. When the inspection is closer range, the evaluation using aerial images can assist in the inspection through image magnification coupled with FLIR thermal image detection and enhanced partial development to understand the status quo. Thus, the tests need to be performed in both static and dynamic ways to analyze the images, among which the majority of the views can be used as the scope and distance of image identification is determined.

If close-range inspection is taken substantially, a great amount of time and labor are needed. Thus, firstly, the infrared thermal imager is used, with the status of external walls or adjacent structures taken into account and the deterioration site recorded. Then the deterioration status of structures is identified by close-range image inspection with an aerial camera. Therefore, the close-range inspection and recording of possibly damaged locations are carried out by the UAV image transmission system, referred to as "image transmission". This technology serves for transmitting the UAV images to the ground wireless image transmission receiving equipment in real time. Generally, the image transmission system consists of three parts: transmitter, receiver and display.

\subsection{Analysis of effective visible distance of UAV image recording}

To verify the UAV's effective visible diatance in the proposed method, a verification test is conducted. The proposed test based on Moore et al. (2018) implements an app named ON 3D Camerameasure which can measure the size of a 3D object using the mobile device's camera; the principle is that if the focal length of the camera and the distance from the object to the camera are known, the length or position of the object to be measured can be measured.

A scaled scale map was designed in this study. The fixed-focus lens configured by the drone was used as a tool for collecting images, and the UAV fixed-focus lens was employed to collect images at different distances. The main purpose of collecting images at a fixed focal length is to reduce the generation of uncertainty. The fixed-focus lens can eliminate the scale conversion after zooming, so the pixel size can be converted according to the distance, just like the parallel line image size. Different distance sizes are used to convert the ratio between the figures and the pixels. In this study, the proposed scale map is used as a reference. The images are collected at a fixed focal length of $5 \mathrm{~m}$ to $10 \mathrm{~m}$, respectively. Based on the scale map or the size of the reference object size, the actual size of each target can be derived, as shown in Figure 4. Based on the test results, the method suggests an effective distance of $5 \mathrm{~m}$ to prevent the UAV's possible impacting the target of the detection.

The proposed test conducted image capturing in a low altitude-shooting mode as a comparison between the actual size and the measurement results and error analysis of

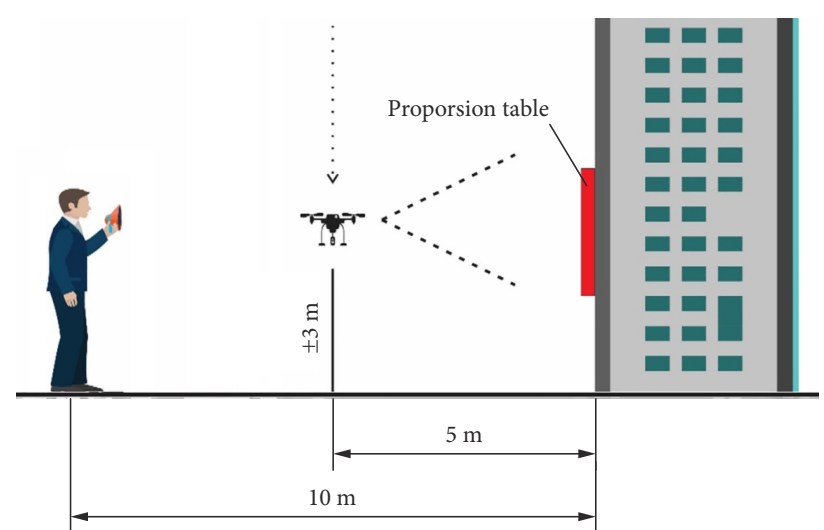

Figure 4. Verification test illustration

the proposed method. This test adopts three test samples named A, B and C. Error values analysis are compared between image measurement and actual measurement. The recorded test results of the analyzed range of error values using this proposed method are shown in Table 1 below.

Table 1. Verification error rate analysis

\begin{tabular}{|c|c|c|c|c|}
\hline $\begin{array}{c}\text { Test } \\
\text { distance }\end{array}$ & Item & $\begin{array}{c}\text { Measuring } \\
\text { size } \\
\text { (unit: } \mathrm{m} \text { ) }\end{array}$ & $\begin{array}{l}\text { Actual size } \\
\text { (unit: } \mathrm{m} \text { ) }\end{array}$ & $\begin{array}{l}\text { Error } \\
\text { rate \% }\end{array}$ \\
\hline \multirow{4}{*}{$5 \mathrm{~m}$} & ratio graph & 0.58 & 6.0 & $3.3 \%$ \\
\hline & Sample A & 1.54 & 1.56 & $1.3 \%$ \\
\hline & Sample B & 2.71 & 2.75 & $1.5 \%$ \\
\hline & Sample C & 3.12 & 3.15 & $1.0 \%$ \\
\hline \multicolumn{4}{|c|}{ Average Error Rate } & $1.8 \%$ \\
\hline \multirow{4}{*}{$10 \mathrm{~m}$} & ratio graph & 0.58 & 6.0 & $3.3 \%$ \\
\hline & Sample A & 1.55 & 1.56 & $0.6 \%$ \\
\hline & Sample B & 2.72 & 2.75 & $1.1 \%$ \\
\hline & Sample C & 3.11 & 3.15 & $1.3 \%$ \\
\hline \multicolumn{4}{|c|}{ Average Error Rate } & $1.6 \%$ \\
\hline
\end{tabular}

\subsection{Analysis of effective visible height of UAV image recording}

Regarding the discussion of the effective visible inspection height, currently all cases chosen are over eight floors high. The fuselage remains stably controlled and the sampled images can be taken through head-up photography and overhead photography. In Figure 5, the remote and timely inspection method added with the high-resolution image can be used to obtain and magnify images, as shown in Figure 6. In addition, by comparing the side-shooting and the real-time UAV inspection, as shown in Figure 7, the proposed detection method can also use the infrared thermal imager to detect the difference in the image, as shown in Figure 8, and then use the UAV image to increase the accuracy of detection position, as shown in Figure 9. By means of real case detection, images are obtained by the image transmission system and information screen- 
shots can be made by smart phone or tablet to facilitate recording. Parameters, such as height, GPS position and azimuth, and real-time images are shown in Figure 10. This study also integrates image comparison technology. By comparing the original CAD with actual detection im-

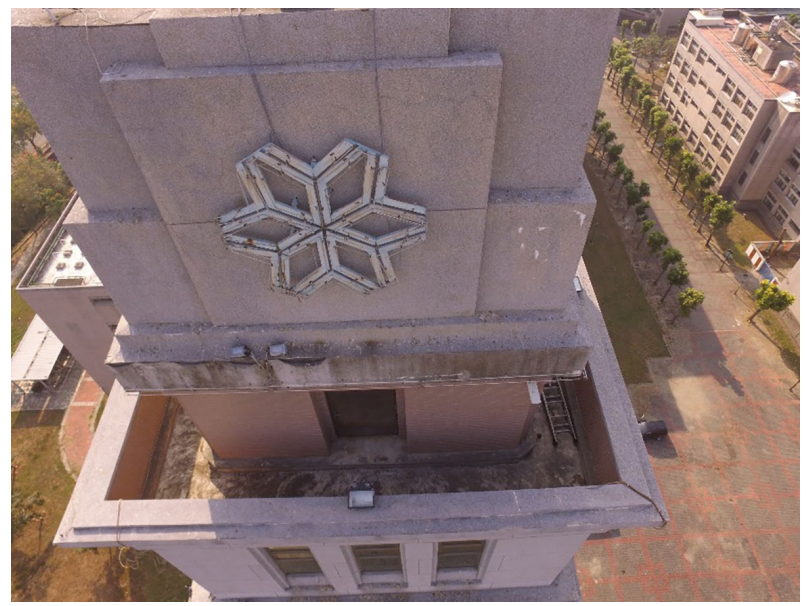

Figure 5. Overhead photography by UAV's camera

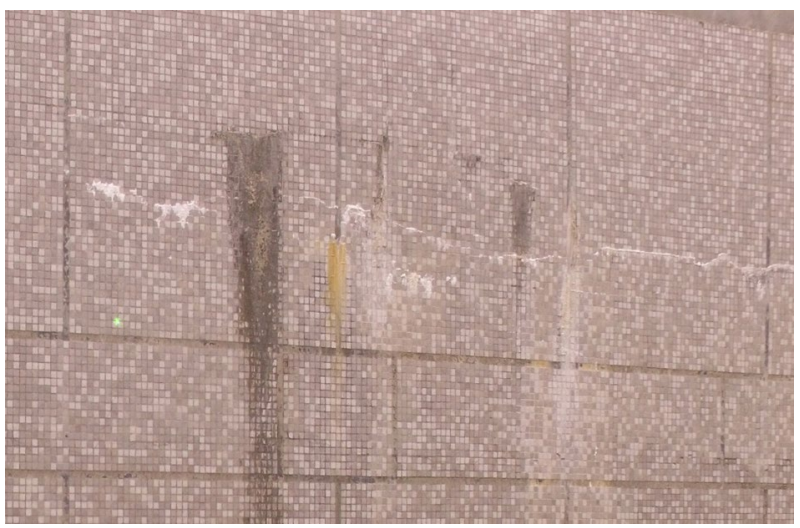

Figure 6. The close-range horizontal photography by UAV's camera

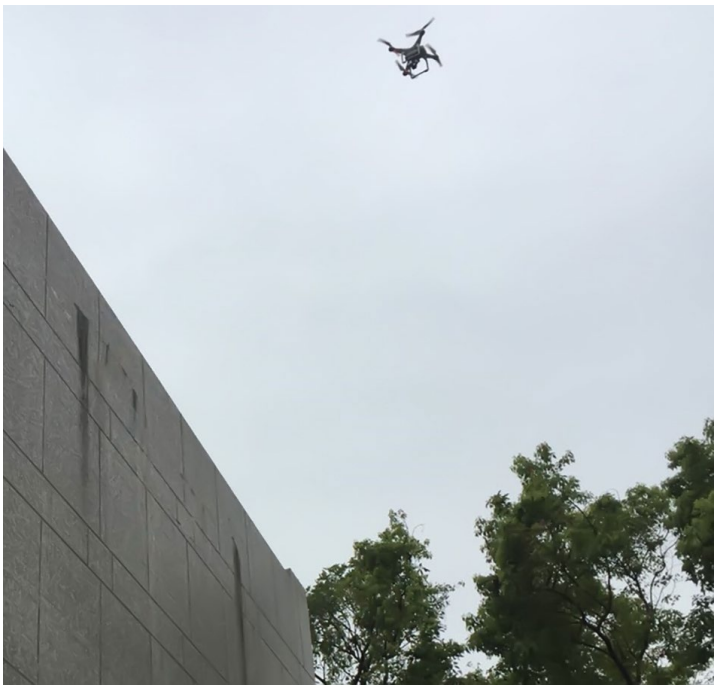

Figure 7. The sample image taken from ground side-shot ages, annotation method in the CAD is adopted to serves as a basis for estimating the size and range of the damaged area and subsequently improving the accuracy and completeness of detection results, as shown in Figures 11(a) and 11(b).

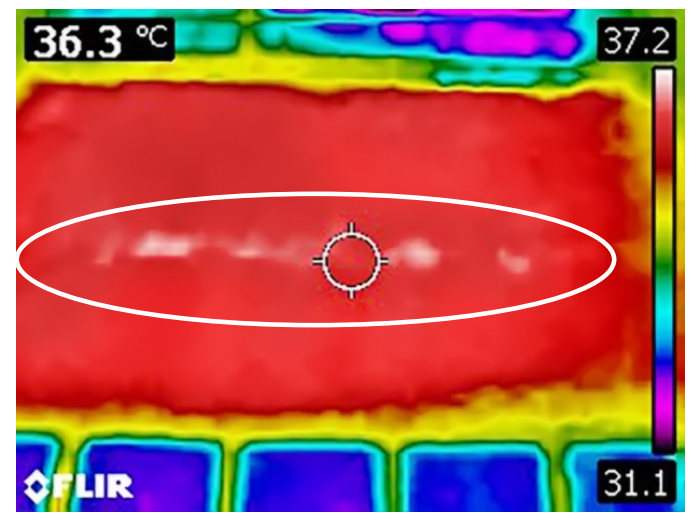

Figure 8 . The sample image taken by FLIR thermal imager

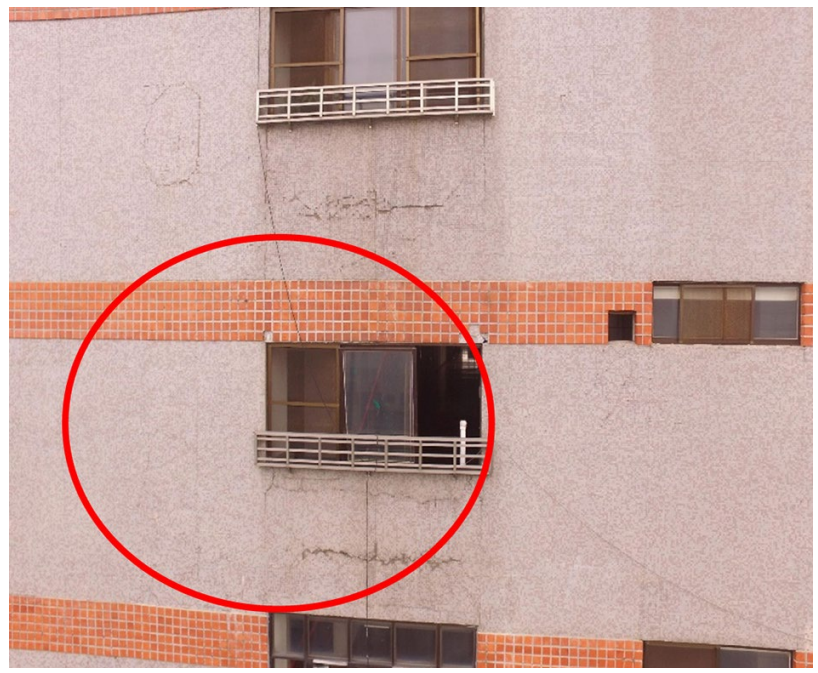

Figure 9. The sample image by UAV

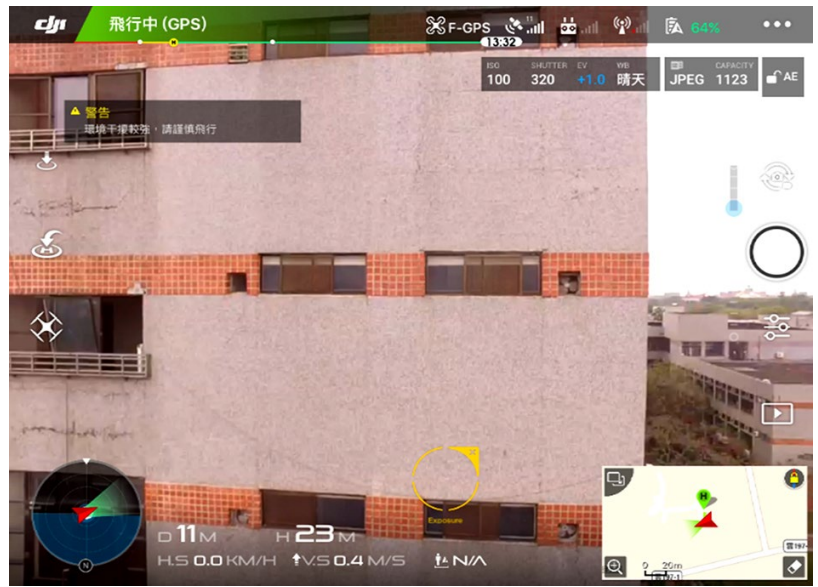

Figure 10. The real time sample picture captured by UAV 


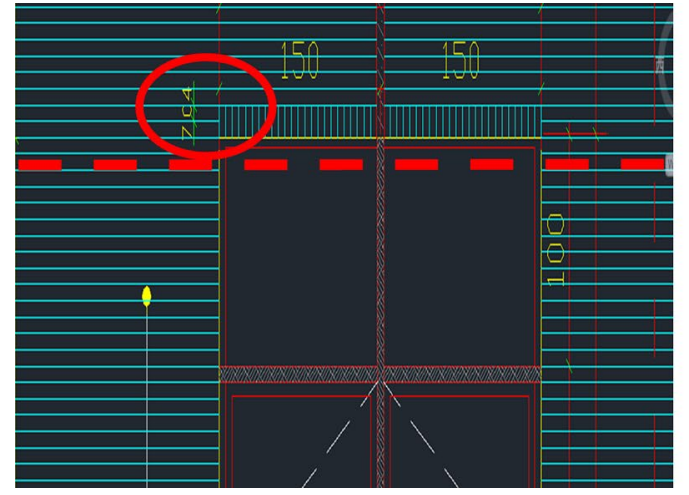

Figure 11(a). The sample of the original CAD

\section{Grading}

In the proposed model procedure, after using the infrared thermal imager to locate possible deteriorated positions, and then employing the UAV to take close-range images, the next step is to grade the degree of damage position for future maintenance strategies. The proposed model defined the deteriorated state as Degree (D), added with high-resolution images from the UAV to visually evaluate the deteriorated area of exterior walls. In addition, according to the deteriorated state of exterior walls or adjacent structures, the common deteriorated types of exterior walls are shown in this study (Table 2).

The deteriorated state is graded on the basis of the images of damage. Grade A represents slight damage, grade $\mathrm{B}$ stands for medium-level damage, grade $\mathrm{C}$ for poor state, and grade $\mathrm{D}$ marks severe damage. The grading clearly reflects the state of the structure. The deteriorated state is estimated through image inspection for the problematic part, and then the structure is graded to show its status. Tai (2008) provided the evaluation tables for each degree of deterioration in his article. Proceeding from Tai's evaluation tables, this study improves the table featuring four

Table 2. Types of exterior walls and causes for deterioration

\begin{tabular}{|l|l|}
\hline \multicolumn{1}{|c|}{$\begin{array}{c}\text { Type of } \\
\text { exterior walls }\end{array}$} & \multicolumn{1}{c|}{ Cause for deterioration } \\
\hline $\begin{array}{l}\text { Metal curtain } \\
\text { wall }\end{array}$ & $\begin{array}{l}\text { The corrosion of metal sheets and the } \\
\text { deterioration of sealants, such as pollution, } \\
\text { discoloration, softness and fading. }\end{array}$ \\
\hline $\begin{array}{l}\text { Tiled exterior } \\
\text { wall }\end{array}$ & $\begin{array}{l}\text { Poor construction, internal and external drop, } \\
\text { concrete crack, rusty window frame rust. }\end{array}$ \\
\hline $\begin{array}{l}\text { Whitening of } \\
\text { red bricks }\end{array}$ & $\begin{array}{l}\text { When the environment remains highly } \\
\text { humid for a long time, these inorganic } \\
\text { compounds can dissolve and re-precipitate } \\
\text { out of the surface, resulting in whitening } \\
\text { exterior. }\end{array}$ \\
\hline Wall cancer & $\begin{array}{l}\text { Preliminary judgment is made through visual } \\
\text { inspection of concrete surface paint peeling } \\
\text { or spalling powder of mortar layer partially } \\
\text { adhered, and the addition of water seepage or } \\
\text { whitening precipitation. }\end{array}$ \\
\hline
\end{tabular}

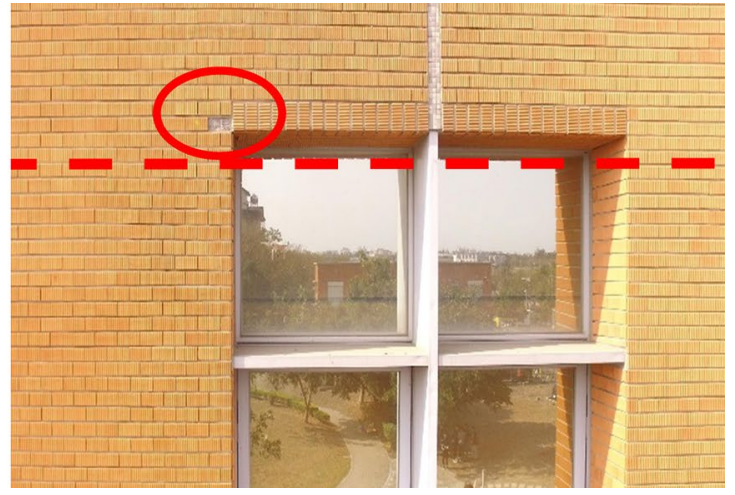

Figure 11(b). The sample image by aerial photography

degrees, as shown in Table 3. In this study, the deteriorated area of exterior walls is estimated by comparing the CAD layer with the images of exterior wall verification, which contributes to evaluating the deteriorated area. This study also provides a comprehensive evaluation method to estimate the deterioration status of exterior walls based on the grading scores of degree and impact given in the evaluation.

\subsection{Web output interface of the proposed system}

This study implements web interface to display the test results of the cases chosen. The information in the proposed web interface contains case ID, test date, the decoration materials' description and detection methods, abnormal description and the evaluations of degree (D) and impact (R). The picture information includes FLIR thermal imager detection images and UAV close-up images, as shown in Figure 12.

According to the test results of Case A, the external wall deterioration is mainly caused by cracks in the magnetic tiles. The obvious temperature difference can be detected by an infrared thermal imager and the inspection images can be obtained by UAV at close range shooting.

Table 3. Degree of deterioration Status

\begin{tabular}{|l|l|}
\hline $\begin{array}{c}\text { Type of } \\
\text { deterioration }\end{array}$ & \multicolumn{1}{c|}{ Degree of deterioration } \\
\hline \multirow{5}{*}{ Stripping } & A. Wall tiles \\
\cline { 2 - 3 } & B. Wall tiles and adhesive layers \\
\cline { 2 - 3 } & C. Composite stripping of substrates \\
\cline { 2 - 3 } & D. Large-area serious stripping \\
\hline \multirow{5}{*}{ Sracking } & A. Grey joints of wall tiles \\
\cline { 2 - 3 } & B. Cracks between wall tiles and adhesives \\
\cline { 2 - 3 } & $\begin{array}{l}\text { C. Cracks in base materials, wall tiles } \\
\text { and bonding materials }\end{array}$ \\
\cline { 2 - 3 } & D. Large-area severe cracks \\
\hline \multirow{5}{*}{ A. Wall tiles } \\
\cline { 2 - 3 } & B. Wall tiles and adhesive layers \\
\cline { 2 - 3 } & $\begin{array}{l}\text { C. Spalling in base materials, wall tiles } \\
\text { and bonding materials }\end{array}$ \\
\cline { 2 - 3 } & D. Wall tiles \\
\hline
\end{tabular}




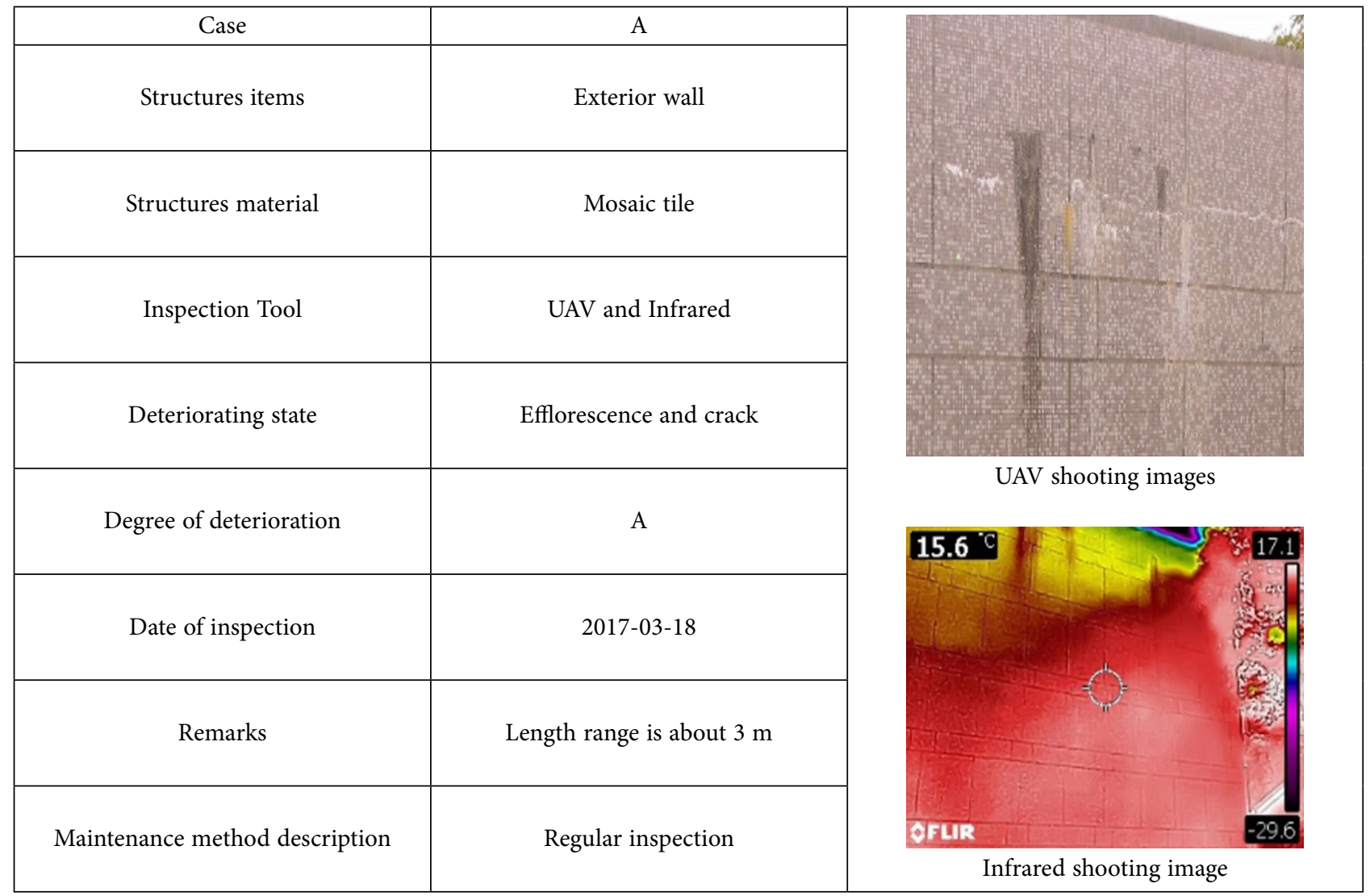

Figure 12. The web interface output of case A

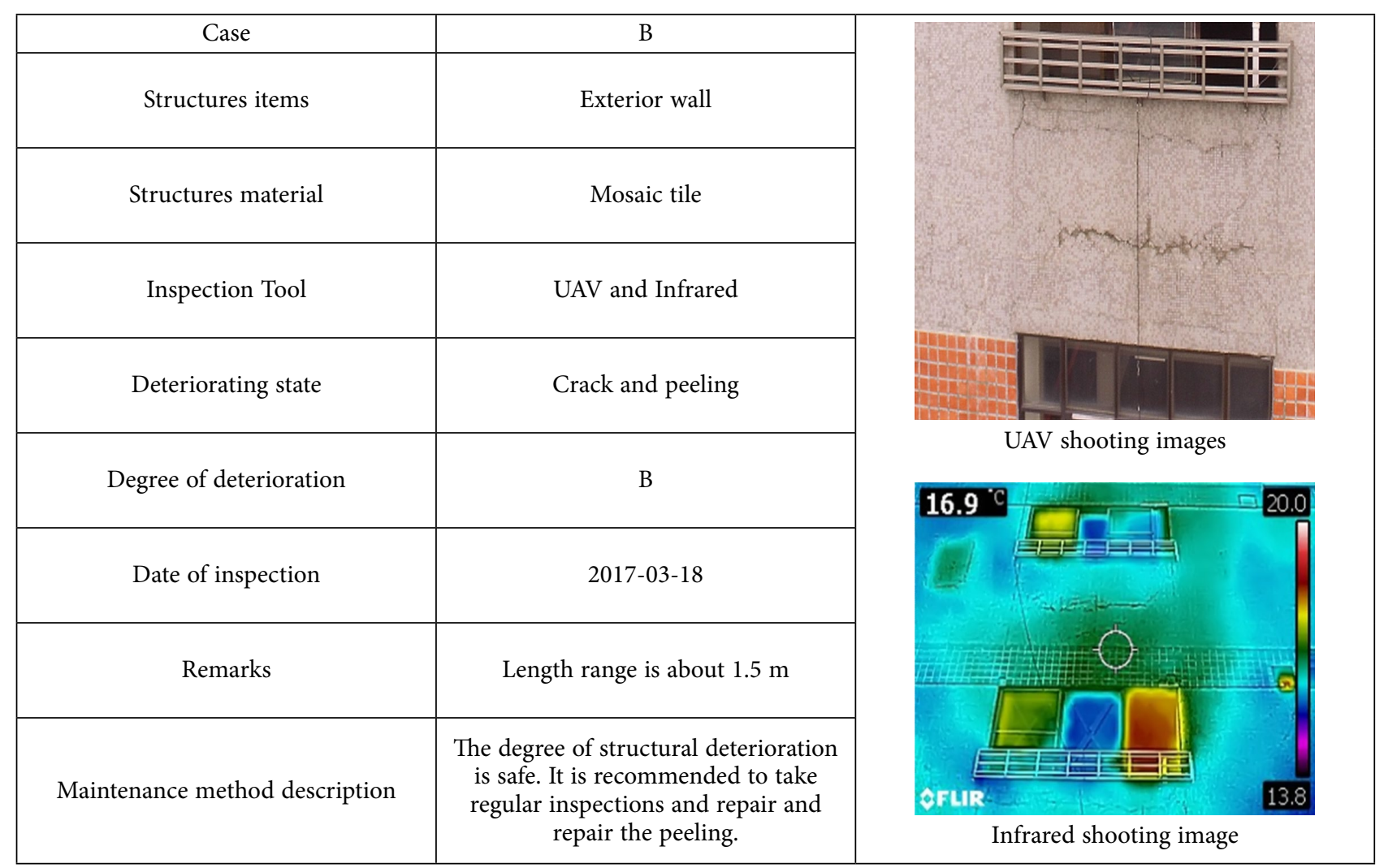

Figure 13. The web interface output of the case B 
In Case $\mathrm{A}$, the image rating reveals that the structure has not been seriously affected. Given that mosaic tiles are small magnetic tiles, regular detection is recommended.

The test results of Case B show there are some cracks and some of the tiles fall off. Using an infrared camera to obtain images, the results reveal that a significant temperature difference around the hunting wind and the crack range can be clearly seen, shown in Figure 13. Then using UAV for close-range flat-view images, we can find that a small number of tiles have fallen off, the crack distribution is at least two, and compound deterioration is occurring. Therefore, the grading result can be $\mathrm{B}$. It means that regular inspection and repair of spalling should be carried out.

\subsection{The findings of the proposed model in cases studies}

The UAV is equipped with highly available and widely used high-resolution photographic equipment, such as a high-quality image recorder. With the maturity of UAV technology and the convenience of operation, the method proposed by this study can locate detection points more effectively and set the relevant height. It can also help to record images in the inspection process, improve the overall visual detection technology, and solve the existing problems of visual detection caused by angle and shelters. The UAV high-resolution photography and stable UAV combined with laser beam are used to enhance image recording because a laser beam can produce uneven images if it encounters bumps or cracks. The comparison of two-dimensional CAD layer images significantly improves the visual interpretation accuracy, overcoming problems caused by top-down view, angle and height. With this proposed method the cost for professional equipment is relatively low, and the exterior wall inspection can reduce accidents and the cost of temporary construction facilities. Through expert questionnaires and consistency analysis, this study summarizes and establishes the current value index of exterior walls or ancillary structures, and the status quo evaluation mode of exterior walls or ancillary structures.

\section{Conclusion and suggestions}

The conclusions are listed as follows:

1. This study presents an improved way that combines the visual inspection method, infrared detection technology, UAV real-time detection, and grading method for evaluating the status of exterior walls for maintenance. Such combination of inspection methods proposed herein has proven cost-effective and safe with case testing compared with the traditional method. In addition, the incorporation of this method into GPS positioning and height recording contributes to locating deteriorated positions and the subsequent reference locations for maintenance. In addition, the parameters setting of the proposed mechanism are analyzed and recommendated in the study.
2. Based on the evaluation criteria of the exterior wall status of middle and high-rise buildings discussed in this study and the current value index of exterior wall or accessory structure, this study establishes the current value evaluation model of exterior wall or ancillary structures.

3. This study proposes an enhancement of detection mechanism for exterior walls or ancillary structures, and an improved way of detection, evaluation and recording, and presents the results of detection by web pages to which users have easy access. The proposed mechanism is more efficient and lower cost for the detection of external walls or ancillary structures' abnormal status, which is easy to use in practice.

4. Case-based reasoning can be introduced in the future. Case databases can be used to retrieve cases of exterior walls or ancillary structures, and data comparison can be used to assist in the application of appropriate maintenance cycle and strategies for renovation. Suggestions for case-based maintenance and strategies for renovation can be developed in a systematic way.

\section{Acknowledgements}

This research was supported by Ministry of Science and Technology of R.O.C. (project ID 107-2622-E-224-003-CC3).

\section{Funding}

This work was supported by the $<$ Ministry of Science and Technology of R.O.C. $>$ under Grant [number 107-2622-E224-003-CC3].

\section{Author contributions}

Nai-Hsin Pan, Ching-Hsiang Tsai, and Jessie Sung have conceived the study and were responsible for the design and structure of the paper. Ching-Hsiang and Tsai KueiYuan Chen performed the experiments of the proposed method and obtained results. Nai-Hsin Pan, Jessie Sung and Ching-Hsiang Tsai wrote drafts of the article. NaiHsin Pan and Jessie Sung are supervisors.

\section{Disclosure statement}

Nothing to declare.

\section{References}

BeckMan, G. H., Polyzois, D., \& Cha, Y. J. (2019). Deep learning-based automatic volumetric damage quantification using depth camera. Automation in Construction, 99, 114-124. https://doi.org/10.1016/j.autcon.2018.12.006

Bošnak, M., Matko, D., \& Blažič, S. (2012). Quadrocopter control using an on-board video system with off-board processing. Robotics and Autonomous Systems, 60(4), 657-667. https://doi.org/10.1016/j.robot.2011.10.009 
Cha, Y. J., Choi, W., \& Buyukozturk, O. (2017). Deep learningbased crack damage detection using convolutional neural networks. Computer-Aided Civil and Infrastructure Engineering, 32(5), 361-378. https://doi.org/10.1111/mice.12263

Cha, Y. J., Choi, W., Suh, G., Mahmoudkhani, S., \& Buyukozturk, O. (2018). Autonomous structural visual inspection using region-based deep learning for detecting multiple damage types. Computer-Aided Civil and Infrastructure Engineering, 33(9), 731-747. https://doi.org/10.1111/mice.12334

Chen, Y., Shen, Y., Liu, X., \& Zhong, B. (2015). 3D object tracking via image sets and depth-based occlusion detection, Signal Processing, 112, 146-153.

https://doi.org/10.1016/j.sigpro.2014.08.046

Chiao, M., \& Kao, Y. W. (2015). Discussion on testing method of bond strength of exterior wall decorative bricks. Sichuan Building Materials, 1, 97-98.

de Freitas, S. S., de Freitas, V. P., \& Barreira, E. (2014). Detection of facade plaster detachments using infrared thermography - A nondestructive technique. Construction and Building Materials, 15, 80-87.

https://doi.org/10.1016/j.conbuildmat.2014.07.094

Edis, E., Flores-Colen, I., \& de Brito, J. (2013). Thermographic inspection of adhered ceramic claddings: Limitation and conditioning factors. Journal of Performance of Constructed Facilities, 6(27), 737-747.

https://doi.org/10.1061/(ASCE)CF.1943-5509.0000365

Engel, J., Sturm, J., \& Cremers, D. (2014). Scale-aware navigation of a low-cost quadrocopter with a monocular camera. Robotics and Autonomous Systems, 62(11), 1646-1656. https://doi.org/10.1016/j.robot.2014.03.012

Fonstad, M. A., Dietrich, J. T., Courville, B. C., Jensen, J. L., \& Carbonneau, P. E. (2013). Topographic structure from motion: a new development in photogrammetric measurement. Earth Surface Processes and Landforms, 38, 421-430. https://doi.org/10.1002/esp.3366

Green, S., Bevan A., \& Shapland, M. (2014). A comparative assessment of structure from motion methods for archaeological research. Journal of Archaeological Science, 46(1), 173-181. https://doi.org/10.1016/j.jas.2014.02.030

Hashim, K. A., Ahmad, A., Samad, Abd. M., Nizam Tahar, K., \& Udin, W. S. (2012). Integration of low altitude aerial \& terrestrial photogrammetry data in $3 \mathrm{D}$ heritage building modelling. In 2012 IEEE Control and System Graduate Research Colloquium, ICSGRC (pp. 225-230), Shah Alam, Malaysia. https://doi.org/10.1109/ICSGRC.2012.6287166

Hehn, M., \& D’Andrea, R. (2014). A frequency domain iterative learning algorithm for high-performance, periodic quadrocopter maneuvers. Mechatronics, 24(8), 954-965. https://doi.org/10.1016/j.mechatronics.2014.09.013

Huang, S. M., Chiang, L. W., \& Chen, C. H. (2010). The method by visual inspection of the building siding public security research. Journal of Property Management, 1(1), 35-44.
Kang, D., \& Cha, Y. J. (2018). Autonomous UAVs for structural health monitoring using deep learning and an ultrasonic beacon system with geo-tagging. Computer-Aided Civil and Infrastructure Engineering, 33(10), 885-902.

https://doi.org/10.1111/mice.12375

Koutsoudis, A., Vidma, B., Ioannakis, G., Arnaoutoglou, F., Pavlidis, G., \& Chamzas, C. (2014). Multi-image 3D reconstruction data evaluation. Journal of Cultural Heritage, 15(1), 73-79. https://doi.org/10.1016/j.culher.2012.12.003

Lee, L. C. (2015). Introduction to infrared camera inspection application. In Taipei Professional Civil Engineering Association, 69, 9-10.

Lee, Y., Liu, Y. F., Chou, H., Bai, L., \& Chiang, W. (2014). Application of infrared thermography to detecting bonding defects of exterior wall decorative bricks. Residence Technology, 1, 52-54.

Lin, K. T. (2014). A feasibility study on infrared thermal imaging technology to inspect defects in exterior wall tile system interface (Master's thesis). Institute of Materials Engineering National Taiwan Ocean University, R.O.C, Taiwan.

Mancini, F., Dubbini, M., Gattelli, M., Stecchi, F., Fabbri, S., \& Gabbianelli, G. (2013). Using unmanned aerial vehicles (UAV) for high-resolution reconstruction of topography: the structure from motion approach on coastal environments. Remote Sensing, 5(12), 6880-6898. https://doi.org/10.3390/rs5126880

Moore, J., Tadonada, H., Kirsche, K., Perry, J., Remen, F., \& Tse, Z. T. H. (2018). Facility inspection using UAVs: a case study in the University of Georgia campus. International Journal of Remote Sensing, 39(21), 7189-7200.

https://doi.org/10.1080/01431161.2018.1515510

Nex, F., \& Remondino, F. (2014). UAV for 3D mapping applications: A review. Applied Geomatics, 6(1), 1-15. https://doi.org/10.1007/s12518-013-0120-X

Tai, P. Y. (2008): Nondestructive testing of exterior wall tiles by tap tone method (Master's thesis). Graduate Institute of Urban Development and Architecture, National University of Kaohsiung, R.O.C., Taiwan.

Technews. (n.d.). http://technews.tw/2015/08/15/drone-cameras/ Turner, D., Lucieer, A., \& Watson, C. (2012). An automated technique for generating georectified mosaics from ultra-high resolution Unmanned Aerial Vehicle (UAV) imagery, based on Structure from Motion (SFM) point clouds. Remote Sensing, 4(5), 1392-1410. https://doi.org/10.3390/rs4051392

Yeh, L. Y. (2014). Technical application of structural health monitoring system for super-tall buildings. Guangdong Architecture Civil Engineering, 2, 62-64.

Yen, J. C. (2013). Testing the degradation of the exterior wall tiles of school buildings by tap tone method (Master's thesis). Department of Civil Engineering, Feng Chia University, R.O.C., Taiwan. 